degree below a Serpentis. When seen on a dark sky it presented an extensive coma with a large bright nucleus and a tail at least half a degree in length. Mr. Franks also reports that it was nearly a degree north of its predicted position on October II, and that the difference is increasing, but it seems probable that he was using the earlier ephemeris published by Dr. Ebell, and not the later one from which we gave an extract last week. The following is a continuation of the corrected ephemeris :-

$$
\begin{array}{rrr}
19 \ldots 15 & 51 \cdot 0 \ldots+14 & 14 \cdot 6 \\
20 \ldots 15 & 52 \cdot 1 \ldots+15 & 8 \cdot 2 \\
21 \ldots 15 & 53 \cdot 1 \ldots+16 & 0 \cdot 6
\end{array}
$$

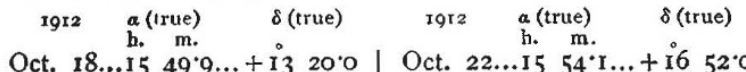

h. $\mathrm{m}$. $23 \ldots 1555 \cdot 1 \ldots+1742 \cdot 5$ $24 \ldots 1556 \cdot 1 \ldots+18 \quad 32 \cdot 0$ $25 \ldots 1557^{\circ} 0 \ldots+1920.5$

According to this ephemeris, the magnitude should now be $6 \cdot 6$, and decreasing slowly, but, as Mr. Franks remarks, the comet is exceeding expectations, and, with its indications of abnormal brightening, may well repay careful observation, especially in the form of a close series of photographs, by those who are favourably situated; on October 2 I the comet will be about one-third of a degree east of $\gamma$ Serpentis.

The Recent Total Eclipse of The Sun.--It is with much regret that we learn from Greenwich that all attempts to make observations of the recent total eclipse of the sun were frustrated by the heavy rain which prevailed in the eclipse region of Brazil on eclipse day, October 1o. The Greenwich observers, Messrs. Eddington and Davidson, were located at Alfenas, an elevated village some $18_{5}$ miles north of Santos, where there were also eclipse parties from France, Germany, Brazil, and other countries. The Brazilian officials rendered all the assistance they could, and the Government voted a sum of $5000 l$. for the reception of the visiting astronomers at Rio. According to a characteristically interesting letter from Mr. J. J. Atkinson, which appeared in The Morning Post on October \&, the Greenwich equipment weighed about three tons, and had to be transported from Rio to the terminus of the State railway, a distance of about I $_{50}$ miles towards the mountains; owing to the sharp incline the latter part of the track has to be worked on the cog system. Mr. Atkinson, who accompanied the Greenwich observers as a volunteer, also recites some interesting reminiscences of his previous eclipse experiences.

The Constant of Aberration.-In No. I5, vol. xxvii., of The Astronomical Journal, Prof. C. L. Doolittle gives the result of twenty-two determinations of the aberration constant derived from thirty-two years' latitude work at the Sayre and Flower Observatories. The observations were made at two different places, with what are practically four different instruments, only the observer remaining the same, and the mean probable error is less than o'or". Taking the weighted mean of all the observations, Prof. Doolittle finds for the constant the value $20^{\circ} 5^{2} 5^{\prime \prime} \pm 0^{\circ} 0043^{\prime \prime}$, and the corresponding value for the solar parallax is $8.780^{\prime \prime}$.

\section{THE AUTUMN MEETING OF THE INSTITUTE OF METALS.}

THE papers presented at the autumn meeting of the Institute of Metals, which took place at the Institution of Electrical Engineers on September 25 and 26 , may be divided into two groups according as their interest lies principally on the practical or on the scientific side.

Among the "practical" group two papers dealing with the joining of non-ferrous metals and alloys may be mentioned. In these Prof. Carnevali, of Turin, and A. E. Tucker, of Birmingham, discuss the question of autogenous welding, although the latter paper also deals in an interesting if somewhat scrappy manner with many other processes, such as soldering and brazing, \&c. In view of the great extension of autogenous welding by means of oxygen and acetylene, the question how far the results of this process can be trusted is an important one. Tucker appears to regard a weld as satisfactory if it is found on testing it to destruction that the fracture occurs away from the weld itself. As a matter of fact, however, the weakest portion of a welded joint, as Carnevali points out, is not the weld itself, but the region of injured metal on either side of it. According to this author the strength of welds in copper and its principal alloys cannot be depended upon, and this conclusion agrees with the views on autogenous welds in iron and steel recently expressed by Fremont and others. In regard to pure aluminium, however, Carnevali finds the method to give satisfactory results, but the efficiency of a weld is much reduced as soon as it is applied to one of the stronger light alloys of aluminium. Broadly speaking, these papers lead one to view the rapid development of autogenous welding practice with some suspicion.

Still on the "practical" side were a number of papers dealing with impurities in copper and copper alloys. An interesting and suggestive paper by E. F. Law dealt with oxygen and oxides as deleterious impurities in alloys. This author took the view that progress in non-ferrous alloys was largely a question of the better elimination of oxides, and this view was strongly supported in the discussion by Rosenhain. The paper by Prof. Turner, however, emphasised the existing difficulties in the way of analytical determination of oxygen in brass, and an appeal was made to chemists to devise a satisfactory method for this purpose. F. Johnson dealt with the effect of impurities, chiefly antimony, on the properties of tough-pitch copper, and here again discussion centred round the part played by oxygen. The lenient view as to the deleterious effects of antimony put forward by the author was, however, strongly opposed by all those who have to deal with copper on the large scale. Other papers of a "practical" character dealt with high-temperature tensile tests on copper and its alloys, and with the annealing of cainage alloys, and both these papers were vigorously criticised in the discussion on the ground of the experimental methods employed by the authors.

The "scientific" papers were not so numerous, but of special interest. Prof. H. C. H. Carpenter contributed two papers dealing in further minute detail with the inversion which he has discovered in a certain range of copper-zinc alloys (brass) at a temperature of $470^{\circ} \mathrm{C}$. In one of these papers the author deals with the effect of impurities on this inversion and finds that any addition of a third metal to these alloys tends to facilitate rather than to inhibit the transformation in question; since the change renders the metal weak and more brittle, it is evident that the use of the purest copper and zinc is desirable in the manufacture of those varieties of brass containing the constituent.

In a very short note Dr. G. T. Beilby, F.R.S., discusses the phenomena of the solidification of metals from the liquid state in reference to the "foam cell" theory of Quincke. In his May lecture to the institute, Dr. Bielby had suggested the importance of a full experimental investigation of the views put forward by Quincke, and the present note is intended to

NO. 2242, VOL. 90] 
state more precisely the problem which Dr. Beilby desires to see investigated. The fundamental question, which goes beyond Quincke's hypothesis, is this, whether the liquid metal undergoes any changes or separations before actual solidification commences, and, if so, whether there is really any formation of foam cells or analogous structures governing the crystallisation of the metal. In his note Dr. Beilby quotes some lines of evidence from the manner in which a thin film of fused salt or other substance solidifies on a glass slip which appears to be strikingly contradictory to Quincke's views, and indeed the impression derived from reading Dr. Beilby's note is rather that he finds the "foam cell" theory less attractive after thus considering it more closely. A practical result is, however, likely to follow from Dr. Beilby's interest in the matter, in the shape of an exhaustive report on our present knowledge of the passage from the liquid to the solid state in metals, prepared under the auspices of a committee of the Institute of Metals, and this will certainly be very welcome.

Of purely theoretical interest is the paper presented by Dr. Rosenhain and. Mr. Ewen, of the National Physical Laboratory, on the intercrystalline cohesion of metals, In this paper the authors advance the hypothesis that the crystals of a pure metal are held together by the action of a thin layer of metal in the amorphous condition forming a species of cement between the crystals. The conception of the existence of such a cement has already been put forward by Bengough and by Osmond, but the authors claim to have used it as a working hypothesis in their own laboratory before others had published their views. The paper begins with a detailed discussion of the general facts which lead in the first place to the idea that there should be some special condition at the boundary surfaces of crystals in solid metals; perhaps the most striking of these facts is the strength of these bounding surfaces, since it has been conclusively shown that pure metals normally undergo fracture through the crystals and not along the boundaries between them; the cohesion across these bounding surfaces is thus stronger than that across the cleavage planes of the crystals themselves.

The authors next suggest in general terms that when two growing crystals approach one another, a region is formed at their boundary in which the molecules are no longer able to assume the crystalline arrangement, and they further point out that if the unit or element of which the crystal is built up is large compared with the "liquid" molecule, then at the junction of two crystals gaps must remain which are too small to contain another complete crystal unit and that consequently such gaps would ultimately be filled by undercooled liquid metal which had been unable to crystallise. This undercooled liquid would then be identical with the "amorphous phase" of Beilby, and would possess similar properties. The paper points out that Beilby has shown that the amorphous phase is more soluble in acids and possesses greater chemical activity than the crystalline phase, and it would accordingly possess a higher vapour pressure under corresponding conditions of temperature. It follows that if two pieces of the same metal, one containing a small and the other a relatively large proportion of amorphous matter, were heated to the same high temperature in a high vacuum, the one containing the larger proportion of amorphous matter would lose weight more rapidly than the other

This conclusion the authors have submitted to the test of experiment in the following manner. If an amorphous intercrystalline cement exists, then a specimen of metal consisting of a few large crystals would contain less of this amorphous material than a piece of the same metal which consists of a very large number of minute crystals, since the material of the cement is chemically identical with the bulk of the metal. Specimens possessing large and small crystals respectively were prepared by the authors in various ways, and these specimens were heated in high vacua ( $0^{\circ} 00_{5} \mathrm{~mm}$. and under) at temperatures sufficient to produce considerable volatilisation losses,

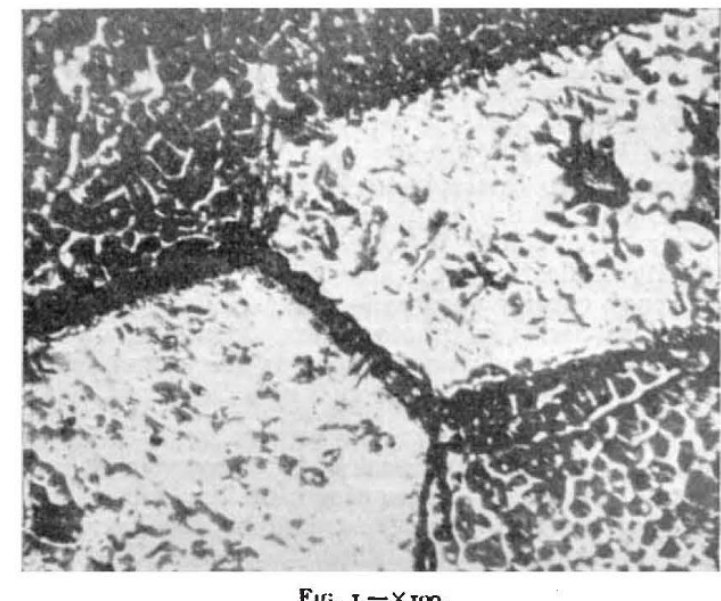

but still well below the melting points of the metals in question.

In the case of silver, zinc, and copper the authors found their expectations verified; the specimens possessing the minute crystal structure in every case losing weight at a greater rate than the coarsely crystalline specimens. The differences found were, in fact, considerably greater than the amount of amorphous cement which could reasonably be supposed to exist in the specimens, but although this point was

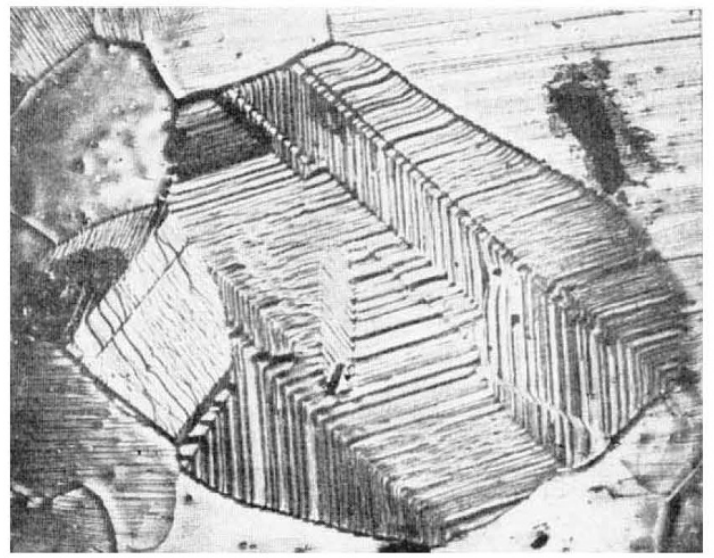

FIG. $2 .-\times 200$.

somewhat laboured in the discussion, the authors offer a fairly satisfactory explanation by showing that the formation of fissures due to the evaporation of the "cement" allows all the crystals in the fine-grained metal to undergo volatilisation from all their surfaces, so that after a time the effective evaporating surface of the fine-grained specimen is much larger than that of the coarse-grained.

This explanation is supported by microscopic evidence, since the widening-by evaporation-of the

No. 2242 , VOL. 90] 
intercrystalline boundaries is clearly visible on the specimens. An example of the channels formed in this way is shown in Fig. I, reproduced from the paper. An interesting confirmation of the authors' views is further found in the tact that while the boundaries between adjacent crystals always exhibit this deep channel, the boundaries of twin-crystals do not show such a groove; since the crystal units on either side of a "twin" boundary fit into one another in a regular manner there is no room for amorphous matter in these boundaries, hence the absence of the groove. With the metals antimony, cadmium, and aluminium the authors obtained irregular losses of weight which neither confirmed nor refuted their views; thev put these views forward merely as a suggestive working hypothesis, and do not claim to have furnished a valid proof of its truth.

Incidentally, the method of heating metals in high vacua furnishes an interesting means of developing their micro-structure, and this method has been used by the authors to study the conditions under which twinned crystals are developed in silver. One of their photomicrographs showing the structure of a twinned crystal of silver is reproduced in Fig. 2 .

\section{THE SURFACE-TENSION OF LIVING CELLS. ${ }^{1}$} PROF. CZAPEK'S pamphlet contains most
important experimental work upon one of the fundamental physical attributes of the living protoplasmic cell, namely, the surface-tension of its external limiting layer. He makes it clear that the tension conditions obtaining in this layer, which intermediates between each metabolic unit and its environment, are of great significance for secretion and for absorption, and he has established that the surface-tension of the cells of the higher plants is maintained fairly constant at the value of about 0.685 , the surface-tension of water in contact with air being taken as unity.

This very important conclusion is the outcome of a line of research which began at an apparently remote point, the successive stages of which may be briefly set out. The work started with the investigation of the curious precipitates that could be produced in the living cells of many plants by the action of dilute ammonia or 0.2 per cent. caffein, such as had been described as "aggregation" by Charles Darwin, in the tentacles of Drosera. Czapek first established that this "myelin-like" precipitate is a compound of caffein with the soluble tannin of the living cell, and is produced in all cells that contain tannin, the mesophyll of Echeveria and Sedum being the most suitable material. He then found that if such living cells are immersed in solutions of organic substances for some hours, the power of giving a precipitate with caffein may be entirely lost. This loss was traced to exosmosis of the tannin from the living cell, and it was further found that for each organic substance there was a particular limiting concentration below which no effect was produced and above which the exosmosis became very rapid. On comparing these limiting concentrations for the series of monovalent alcohols it was found that at each step in the homologous series the molecular concentration required diminished to one-third. Such a relation was, however, exactly what Traube had established for the surface-tension effect of the members of this series.

1 "Ueber eine Methode zur direkten Restimmung der Oberfläcbenspannung der Plasmahaut von Pflanzenzellen." By F. Czapck. Pp. iv +86 . (Jcna: Gustav Fischer, 1911). Price 2.60 marks.

NO. 2242 , VOL. 90]
Following up this clue, Czapek measured the surface-tension of a large number of solutions of organic substances, and compared their action in causing exosmosis from the cell with their activity in lowering the surface-tension of water. He thus was able to establish securely the unexpected generalisation that on dissolving in water sufficient of any organic substance whatever to lower the surfacetension to about 0.685 , a solution is obtained which just causes the exosmosis of the contents of living cells. The critical concentration may require twelve to twenty-four hours to produce its effect, but stronger solutions with a lower surface-tension work very quickly and thoroughly, so that after a short time treatment of the cells with caffein gives no intravital precipitate at all.

The power of the protoplast to retain its dissolved contents is thus shown to be a matter of physical organisation, depending upon the surface-tension of the cell being below that of the medium in contact with its outer surface.

From true solutions Czapek passed to try the effect of emulsion-colloids of a lipoid nature (proteids and carbohydrates do not lower surface-tension enough to give the critical value of 0.685 ). The lipoid emulsions are, however, extremely active, and give exactly the same effects as true solutions.

The last step in the progress was an attempt at identification of the substance actually present in the plasmatic membrane which causes it to have normally so low a surface-tension as $0 \cdot 685$. Czapek finds that saturated emulsions of neutral fats lower the surfacetension just to this value and no further, so that it seems very probable that these are the effective substances in the living cell.

We have thus striking support for the view, widely adopted from the work of Overton and Meyer, that the plasmatic membrane is of a lipoid nature. Overton's later view was that lecithin and cholesterin rather than neutral fats were the particular lipoids present, but these give a lower surface-tension down to about 0.5 . The present line of work indicates that these may be the effective substances in some cells, not those of the higher plants, for yeast and red-blood corpuscles require a medium of about this lower surface-tension to bring on exosmosis of invertase and hæmoglobin respectively.

Many supporters of the lipoid theory of the constitution of the plasmatic membrane have interpreted it to mean that there exists at the surface of the cell a continuous film of a lipoid nature, and this has raised difficulties in understanding the intake of typical nutrient substances which are freely soluble in water, but not in fat. Czapek points out that an emulsion containing only a small percentage of fat is all that is needed to endow the cell with the observed specific properties.

Willard Gibbs showed from thermodynamical considerations that substances in a solution which strongly reduce surface-tension must accumulate in the surface-layer until their return by local excess of osmotic pressure produces a state of equilibrium between the surface and the mass. With emulsified fat particles, however, the osmotic pressure is very slight, and very great accumulation in the surface-layer must result.

This piece of work may serve as a model of scientific method on account of the way in which the mysterious phenomenon of " aggregation," described by Charles Darwin, has been followed on and on until it has led to the evaluation of so fundamental a vital constant as the surface-tension of the living cell. 